perceived in the implementation of successful mitigation strategies. Data was later utilized for descriptive analysis.

The survey was sent to 317 participants, obtaining 71 answers (22,3\% response rate). $23 \%$ of the respondents where pediatric residents. $84,5 \%$ consider that managing pain related to MPP is relevant and must be addressed in every patient. The procedures perceived as most painful (scale from 1 to

10) were arterial punctures (median 9), followed by lumbar punctures and venous punctures (median 7 both). The entire sample declared knowing at least one measure to reduce pain related to MPP, where the most common were: distraction $(84,5 \%)$ non-nutritious suctioning $(78,9 \%)$ and topical creams (76\%). Still, $15,5 \%$ of respondents routinely do not use any measure. $40 \%$ declare that during their training they were taught that pain related to MPP should always be managed in a multimodal way, existing effective measures available. 94,4\% of respondents believe that there are consequences regarding this type of pain; yet, half of the respondents agree that consequences are minimal and last only for a short period of time. The main barriers against implementation identified are a lack of knowledge of existing available measures $(78,9 \%)$ and a belief that these require additional time (60,6\%).

There is concern among respondents regarding the need for adequate management of this type of pain. In spite of this, a large proportion of participants do not routinely use any measure. This might be explained by an underestimation of the important consequences of this type of pain. The low response rate obtained is one of the main limitations of the study. The findings of this survey could assist in planning better and more effective strategies to support the use of effective measures to reduce pain related to MPP among pediatricians.

\section{OSTEOMYELITIS MIMICKING AS NON-ACCIDENTAL INJURY IN EX PREMATURE INFANT}

A Kaninde*, M Wayland, M Chomicki, R Ramaswamy. Peterborough City Hospital, UK

10.1136/archdischild-2021-europaediatrics.40

Introduction Non-Accidental injury (NAI) in children is an important cause of major injury, morbidity and mortality in children. Often times posing as diagnostic challenge to differentiate from underlying pathological cause[1-3]. The Trauma Audit Research Network (TARN) report 'Severe injury in Children 2012', identified that children under the age of 2 years who suffered significant trauma, were found to have NAI as the root cause. Out of those, the majority of cases with suspected NAI $(76 \%)$ were less than 1 year of age[4]

Case Summary We report a case of 33 weeks to immigrant family, presenting to Accident and Emergency department at 7 weeks of age with painful movement of left shoulder. Mother gave history of hearing a 'pop' while changing his vest on day of presentation. Due to language barrier, there was delay in seeking medical help.

Due to delay in seeking help and implausible mechanism of injury, a suspicion of non-accidental Injury was raised and a child protection medical examination was performed.

On examination, it was noted that he had painful movements of left shoulder joint with asymmetrical Moro's reflex. There were no other physical injury marks or bruises.
$\mathrm{X}$ ray of left shoulder was reported as indicative of possible periosteal elevation of left mid-shaft humerus with a recommendation to consider further imaging.

On second day of admission, patient had low grade pyrexia prompting septic screen. On further imaging, MRI shoulder was performed revealing changes suggestive of osteomyelitis.

His initial inflammatory markers were normal. Diagnosis of osteomyelitis was confirmed based on temperature spikes, MRI finding and clinical improvement in range of movements in left arm after starting on intravenous antibiotics.

Discussion Considering a diverse spectrum of presentation for child abuse, it can throw diagnostic challenges to any clinician to differentiate between a pathological cause and Non accidental Injury[3].We had to consider brachial plexus injury, Cerebral palsy as differentials as patient was afebrile initially. It demonstrates how close; range of differentials could be. In past, there are anecdotal example of Neonatal osteomyelitis presenting as Non accidental injury[5].

Prematurity, socio- economic background are independent risk factors contributing to NAI[6]. In one of the American studies, they described NAI doubles risk of mortality by twofold [7], so one should be very vigilant in dealing with cases of suspected NAI

Conclusion Our case highlights importance of non-judgemental and neutral approach while dealing with cases of suspected NAI.

\section{RISK OF OBESITY AND INFANT FEEDING PRACTICES: RESULTS OF PARENTAL SURVEY}

Vitalii Kukushkin*, Olena Starets, Natalia Kotova. Odesa National Medical University

10.1136/archdischild-2021-europaediatrics.41

Overweight and obesity in children seem to be quite common problem in many countries. The food dominants and preferences are developing in early childhood. Not rationale feeding of infants and their overweight are risk factors for obesity in school age. The goal of study was to assess the physical development and feeding practice of infants based on self reported parental survey.

The anonymous parental survey for parameters of physical development and feeding practice details was done randomly. The parents of 2 nd - 3rd year old children were asked to complete special Google-forms. The individual data, overall tables and diagrams were obtained on-line.

Totally 168 filled questionnaires were processed. The data for body weight in the age of 1 year (z-score) shown that $22,6 \%$ children had weight $>+2 \sigma ; 1,8 \%$ children had weight $>+3 \sigma$. For body length it was revealed that $8,9 \%$ children had parameter $>+3 \sigma$. The weight/length indicator for $8,9 \%$ children was $>+2 \sigma$; for $3,0 \%$ children $>+3 \sigma$. The natural feeding since birth was started for $88,1 \%$ of children; until 6 months $61,9 \%$ of children received breast milk, until 12 months $-54,8 \%$. From those children who received breast milk 35,1\% were given also a water during first months. The practice of scheduled feeding followed in 19,1\% families. The first solid food in the age of 4-6 months was administered in $91,6 \%$ of children; mostly presented by vegetable pure. Part $(49,6 \%)$ of children received a meat since the age of 7 months. In the age of 9-12 months feeding of infants was quite diverse. But many parents gave to babies food or drinks 